\title{
ON THE EXISTENCE AND UNIQUENESS OF SOLUTIONS TO A SINGULAR NONLINEAR BOUNDARY VALUE PROBLEM ARISING IN ISOTHERMAL AUTOCATALYTIC CHEMICAL KINETICS
}

\author{
by D. J. NEEDHAM and A. C. KING
}

(Received 15th January 1992)

In this paper we consider the questions of existence and uniqueness of solutions to a singular, nonlinear boundary value problem arising from a model problem in isothermal autocatalytical chemical kinetics. The boundary value problem occurs in the construction of a small time asymptotic solution to an initial-boundary value problem (King and Needham [14]), and existence and uniqueness for the boundary value problem are required for consistency of this formal asymptotic solution.

1991 Mathematics subject classification: 34

\section{Introduction}

In a one-dimensional unstirred environment, the study of the isothermal autocatalytic reaction scheme,

$$
A \rightarrow B \quad \text { rate }=k[A][B]^{p},
$$

(where $A, B$ are reactant and autocatalyst respectively, $k>0$ is the rate constant and $p>0$ in the reaction order) leads to an examination of the coupled reaction-diffusion initial-boundary value problem,

$$
\begin{gathered}
\frac{\partial \alpha}{\partial t}=\frac{\partial^{2} \alpha}{\partial x^{2}}-\left(\alpha \beta^{P}\right)_{+}, \quad \frac{\partial \beta}{\partial t}=\frac{\partial^{2} \beta}{\partial x^{2}}+\left(\alpha \beta^{p}\right)_{+}, \quad x, t>0 \\
\alpha(x, 0)=1, \quad x \geqq 0, \quad \beta(x, 0)= \begin{cases}g(x), & 0 \leqq x \leqq \sigma, \\
0, & x>\sigma,\end{cases} \\
\alpha_{x}(0, t)=\beta_{x}(0, t)=0, \quad t>0
\end{gathered}
$$




$$
\alpha(x, t) \rightarrow A(t), \beta(x, t) \rightarrow B(t) \text {, with } \quad 0 \leqq A(t) \leqq 1,0 \leqq B(t)<\infty \quad \text { as } \quad x \rightarrow \infty, t>0 .
$$

Here $\alpha(x, t), \beta(x, t)$ are dimensionless concentrations of the reactant and autocatalyst respectively, $x$ is dimensionless distance and $t$ is dimensionless time, with the notation $\left(\alpha \beta^{P}\right)_{+}$defined to be,

$$
\left(\alpha \beta^{P}\right)_{+}= \begin{cases}0, & \alpha \leqq 0 \text { or } \beta \leqq 0 \\ \alpha \beta^{P}, & \alpha, \beta>0\end{cases}
$$

In (1.3), $g(x)>0$ is an analytic function in $0 \leqq x \leqq \sigma$, and so $g(x) \sim g_{\sigma}(\sigma-x)^{r}$ as $x \rightarrow \sigma^{-}$, for some constant $g_{\sigma}>0$ and $r \in \mathbb{N}$. Under these conditions it is readily shown (via the scalar maximum principle for parabolic operators) that $\alpha(x, t), \beta(x, t) \geqq 0$ for all $x, t>0$.

For $p \geqq 1$ the initial-boundary value problem (1.2)-(1.5) has been studied extensively by Merkin et al. [19], Merkin and Needham [16,17,18], Gray et al. [11], Billingham and Needham $[4,5,6,7]$ and Needham and Merkin [21]. An important part of examining this system is a full understanding of the scalar initial-boundary value problem,

$$
\left.\begin{array}{c}
\frac{\partial u}{\partial t}=\frac{\partial^{2} u}{\partial x^{2}}+\left(u^{P}\right)_{+}, \quad x, t>0, \\
u(x, 0)=\left\{\begin{array}{ll}
g(x) ; & 0 \leqq x \leqq \sigma \\
0 ; & x>\sigma,
\end{array}\right\} \\
u_{x}(0, t)=0, \quad t>0,
\end{array}\right\}
$$

$u(x, t) \rightarrow u_{\infty}(t)$, with $0 \leqq u_{\infty}(t)<\infty$, as $x \rightarrow \infty, t>0$.

Here $(\cdot)_{+}$is defined as in (1.6) and throughout the paper, the notation $(\cdot)_{+}$will have the following definition,

$$
(f(x))_{+} \equiv \begin{cases}f(x), & x \geqq 0 \\ 0, & x<0 .\end{cases}
$$

We will refer to the above problem as $I[p]$. With $p \geqq 1, I[p]$ has been studied extensively (see, for example, Fujita [10], Bandle and Levine [1], Weissler [24], Levine [15]). For $0<p<1$, the equivalent "sink" problem (with $+(u p)_{+}$replaced by $-(u p)_{+}$in $I[p])$ has been considered in detail by Bandle and Stakgold [2] and Grundy and Peletier [12]. The corresponding source problem $I[p]$ has recently been examined by King and Needham [14] and Needham [20], who in particular obtain an asymptotic solution to $I[p]$ as $t \rightarrow 0^{+}$, uniform in $x$, using the method of matched asymptotic expansions. In the course of the analysis in [14] the following modified initial-boundary value problem arose, 


$$
\left.\begin{array}{rl}
\frac{\partial U}{\partial t}=\frac{\partial^{2} U}{\partial \bar{X}^{2}}+\left(U^{P}\right)_{+}, \quad-\infty<\bar{X}<\infty, \quad t>0, \\
U(\bar{X}, 0)=\left\{\begin{array}{ll}
g_{\sigma}(-\bar{X})^{2 /(1-p)}, & \bar{X}<0, \\
0 & , \quad \bar{X} \geqq 0
\end{array}\right\} J[p] \\
U(\bar{X}, t) \rightarrow\left\{\begin{array}{lll}
(1-p)^{1 /(1-p)} t^{1 /(1-p)} & \text { as } \quad \bar{X} \rightarrow \infty \\
g_{\sigma}(-\bar{X})^{2 /(1-p)} & \text { as } & \bar{X} \rightarrow-\infty
\end{array}, \quad t>0,\right.
\end{array}\right\}
$$

Following [14], this problem is reduced by the similarity transformation,

$$
U(\bar{X}, t)=t^{1 /(1-p)} V(X),
$$

with $X=\bar{X} t^{-1 / 2}$. On substituting from (1.7) into $J[p]$, we are left with the following nonlinear boundary value problem for $V(X)$, namely,

$$
\begin{aligned}
V_{X X}+\frac{1}{2} X V_{X}+\left[V^{p}-\frac{1}{1-p} V\right]_{+}=0, & -\infty<X<\infty, \\
V(X) & \geqq 0 \text { for all }-\infty<X<\infty \\
V(X) & \rightarrow \begin{cases}(1-p)^{1 /(1-p)}, & X \rightarrow+\infty \\
g_{\sigma}(-X)^{2 /(1-p)}, & X \rightarrow-\infty\end{cases}
\end{aligned}
$$

which will henceforth be referred to as BVP. It should be noted that in the reduction of $J[p]$ to BVP, we have replaced $\left(V^{p}\right)_{+}-(1 /(1-p)) V$ by $\left[V^{p}-(1 /(1-p)) V\right]_{+}$. This is allowable by condition (1.9) and will be convenient in what follows. The details of this problem were not considered in [14]; only the asymptotic forms as $X \rightarrow \pm \infty$, which were immediately required as matching conditions, were derived. However, for the asymptotic structure derived in [14] to be formally complete, we require that for a fixed $0<p<1$, then BVP has a unique solution for each $g_{\sigma}>0$. It is this existence and uniqueness question for BVP which we consider in the present paper. Related problems in the non-singular case $p>1$ have been considered by Escobedo and Zua Zua [9].

We adopt a shooting method, similar in spirit to that used by Berestycki et al. [3] and Peletier and Serrin [22] for radial problems on the half line. This method is adapted for BVP, which is defined on the full line. In particular, we consider a modified boundary value problem $\overline{\mathrm{BVP}}$ (defined in (2.1)-(2.4)) for $u=\hat{u}(X),-\infty<X<\infty$ and establish the following main theorem.

Theorem. The set of solutions to $\overline{\mathrm{BVP}}$ consists of a one-parameter family, which can 
be parametrized by $\delta>0$. For each $\delta>0 \exists$ a unique solution of $\overline{\mathrm{BVP}}$ if and only if $v=v_{c}(\delta)$. Moreover, that solution can be constructed in terms of solutions to IVP1,2 as

$$
\hat{u}(X)= \begin{cases}\tilde{u}\left(X, v_{c}(\delta)\right), & x \geqq 0 \\ \bar{u}\left(X, v_{c}(\delta)\right), & x<0\end{cases}
$$

Here $\tilde{u}$ and $\bar{u}$ are solutions of the initial value problems IVP1,2, (defined in $(3.1,2)$ and $(4.1,2)$ respectively) with $v_{c}(\delta)$ being a critical value of $v$ defined in section 3 . This theorem enables existence and uniqueness for BVP to be deduced directly.

\section{A modified boundary value problem}

We consider in this section a modified form of BVP, namely,

$$
\begin{gathered}
U_{X X}+\frac{1}{2} X U_{X}+\left[U^{p}-\frac{1}{1-p} U\right]_{+}=0, \quad-\infty<X<\infty, \\
U(X) \geqq 0 \text { in }-\infty<X<\infty \\
U(X)=0\left[(-X)^{2 /(1-p)}\right] \text { as } X \rightarrow-\infty \\
U(X) \rightarrow(1-p)^{1 /(1-p)} \text { as } X \rightarrow+\infty
\end{gathered}
$$

which we will refer to as $\overline{\mathrm{BVP}}$. A solution of $\overline{\mathrm{BVP}}$ is to be a solution in the classical sense; that is, a twice continuously differentiable function $U(X)$ satisfying (2.1) on $-\infty<X<\infty$, together with conditions $(2.2)-(2.4)$. We begin by first establishing some general properties concerning $\overline{\mathrm{BVP}}$.

Proposition 2.5. Let $U(X)$ be a solution of equation (2.1) in a neighbourhood $N_{0}$ of $X=X_{0}$, such that at $X=X_{0}, U\left(X_{0}\right)=U_{X}\left(X_{0}\right)=0$, whilst $U(X) \geqq 0$ in $N_{0}$; then,

(i) $X_{0}>0 \Rightarrow U(X)=0$ in $X \geqq X_{0}$

(ii) $X_{0}<0 \Rightarrow U(X)=0$ in $X \leqq X_{0}$

(iii) $X_{0}=0 \Rightarrow U(X)=0$ for all $-\infty<X<\infty$.

Proof. (i) In this case $X_{0}>0$ and $U\left(X_{0}\right)=U_{X}\left(X_{0}\right)=0$, with $U(X) \geqq 0$ in $N_{0}$. For $X \in N_{0}$, we now multiply (2.1) by $U_{X}$ and apply $\int_{X_{0}}^{X} \ldots d s$, to obtain

$$
U_{X}^{2}(X)=-\int_{X_{0}}^{X} s U_{s}^{2}(s) d s-\frac{2 U^{p+1}(X)}{(1+p)}+\frac{U^{2}(X)}{(1-p)} ; \quad X \in N_{0}
$$

after use of the conditions at $X=X_{0}$. We now take $X>X_{0}$, and use the mean-value theorem on the first term on the right-hand side of $(2.5)$, to give, 


$$
U_{X}^{2}(X)=-\left(\dddot{X}-X_{0}\right)\left[\hat{X} U_{X}^{2}(\hat{X})\right]-\frac{2 U^{p+1}(X)}{(1+p)}+\frac{U^{2}(X)}{(1-p)} ; \quad X \in N_{0}
$$

with $\hat{X} \in\left(X_{0}, X\right)$. Now, since $0<p<1$, there exists a $\delta>0$, depending upon $X_{0}$ and $p$, such that, from (2.6), $U_{X}^{2}(X) \leqq 0 \forall X \in\left[X_{0}, X_{0}+\delta\right]$. Hence $U_{X}(X) \equiv 0$ in $\left[X_{0}, X_{0}+\delta\right]$, and as $U\left(X_{0}\right)=0$ and $U(X)$ is continuous in $N_{0}$, we conclude that $U(X) \equiv 0$ for $X \in\left[X_{0}, X_{1}\right]$ for any $X_{1}>X_{0}$, and the results follow.

Parts (ii) and (iii) are established similarly.

This result can be used to establish the following monotone property for all solutions to $\overline{\mathrm{BVP}}$.

Proposition 2.7. Let $U(X)$ be a solution of $\overline{\mathrm{BVP}}$, then $U(X)$ is strictly monotone decreasing, with $U(X)>(1-p)^{1 /(1-p)}$ for all $-\infty<X<\infty$.

Proof. From condition (2.2), $U(X) \geqq 0$. However, conditions (2.2)-(2.4) together with Proposition 2.5 lead us to conclude that $U(X)>0$ for all $-\infty<X<\infty$. Now, suppose that $U(X) \geq(1-p)^{1 /(1-p)}$ for all $-\infty<X<\infty$, then (via conditions (2.3), (2.4)) $U(X)$ must have a local minimum at $X=X_{T}$ (say), with $0<U\left(X_{T}\right)<(1-p)^{1 /(1-p)}, U^{\prime}\left(X_{T}\right)=0$ and $U^{\prime \prime}\left(X_{T}\right) \geqq 0$. However, using equation $(2.1)$ we have $U^{\prime \prime}\left(X_{T}\right)=(1 /(1-p)) U\left(X_{T}\right)-$ $U^{P}\left(X_{T}\right)<0$, which gives a contradiction. Hence $U(X) \geqq(1-p)^{1 /(1-p)}$ for all $-\infty<X<$ $\infty$. Next suppose that $U(X)$ has a turning point at $X=\bar{X}_{T}$ (say), then, via (2.1),

$$
U^{\prime \prime}\left(\bar{X}_{T}\right)=\frac{1}{1-p} U\left(\bar{X}_{T}\right)-U^{P}\left(\bar{X}_{T}\right) \begin{cases}>0, & U\left(\bar{X}_{T}\right)>(1-p)^{1 /(1-p)} \\ =0, & U\left(\bar{X}_{T}\right)=(1-p)^{1 /(1-p)}\end{cases}
$$

Thus, $U(X)$ can only have a local minimum for all $-\infty<X<\infty$. It then follows from conditions (2.3), (2.4) that $U(X)$ must be strictly monotone decreasing in $-\infty<X<\infty$, after which the above inequality tightens to $U(X)>(1-p)^{1 /(1-p)}$ for $-\infty<X<\infty$, as required.

These results will be revisited at a later stage. We now adopt a shooting technique to obtain the complete family of solutions to BVP. This involves the study of two related initial value problems.

\section{The initial value problem in $X>0$}

In this section we consider the initial value problem,

$$
\begin{gathered}
\tilde{u}_{X X}+\frac{1}{2} X \tilde{u}_{X}+\left[\tilde{u}^{p}-\frac{1}{1-p} \tilde{u}\right]_{+}=0, \quad X>0 \\
\tilde{u}(0)=(1-p)^{1 /(1-p)}+\delta, \quad \tilde{u}_{X}(0)=-v \delta
\end{gathered}
$$


where $\delta \geqq 0$ and $v \geqq 0$, and we henceforth refer to this as IVP1. We first make the following remark.

Remark 3.3. With $\delta=0$, IVP1 clearly has the global solution $\tilde{u}(X) \equiv$ $(1-p)^{1 /(1-p)} \forall X \geqq 0$. It follows that this is unique through an application of the local uniqueness result (Coddington and Levinson, [8, Theorem 2.2]).

We now restrict attention to the case when $\delta>0$, and to proceed, we require the corresponding linearized initial value problem, namely,

$$
\begin{gathered}
u_{l X X}+\frac{1}{2} X u_{l X}-\left[u_{l}-(1-p)^{1 /(1-p)}\right]=0, \quad X>0 \\
u_{l}(0)=(1-p)^{1 /(1-p)}+\delta, \quad u_{l X}(0)=-v \delta
\end{gathered}
$$

which we shall refer to as LIVP1. The general solution to equation (3.4) is readily obtained, and conditions $(3.5,6)$ determine the unique solution to LIVP1 as,

$$
u_{l}(X)=(1-p)^{1 /(1-p)}+\delta v\left(1+\frac{1}{2} X^{2}\right) \int_{X}^{\infty} \frac{e^{-s^{2} / 4}}{\left(1+\frac{1}{2} s^{2}\right)^{2}} d s+\delta(1-\sqrt{\pi} v)\left(1+\frac{1}{2} X^{2}\right),
$$

for all $X \geqq 0$. We note that for $X \gg 1$,

$$
u_{l}(X) \sim(1-p)^{1 /(1-p)}+\frac{2 \delta v e^{-X^{2} / 4}}{X\left(1+\frac{1}{2} X^{2}\right)}+\frac{\delta}{2}(1-\sqrt{\pi} v) X^{2}
$$

For $v=1 / \sqrt{\pi}, u_{l}(X)$ is monotone decreasing in $X$ with $u_{l}(X) \rightarrow(1-p)^{1 /(1-p)}$ as $X \rightarrow \infty$ However, for $0 \leqq v<1 / \sqrt{\pi}, \quad u_{l}(X)>(1-p)^{1 /(1-p)}$ for all $X>0$ and $u_{l}(X) \sim$ $(\delta / 2)(1-\sqrt{\pi} v) X^{2}$ as $X \rightarrow \infty$. For $v>1 / \sqrt{\pi}, u_{l}(X)$ is monotone decreasing with $u_{l}(X) \sim$ $-(\delta / 2)(\sqrt{\pi \nu}-1) X^{2}$ as $X \rightarrow \infty$. We are now able to relate $\tilde{u}(X)$ to $u_{l}(X)$.

Proposition 3.9. Let $\tilde{u}(X)$ be a solution to IVP1 for $X \in\left[0, X_{e}\right]$ for any $X_{e}>0$. Then $\tilde{u}(X) \geqq u_{l}(X)$ and $\tilde{u}_{X}(X) \geqq u_{l X}(X) \forall X \in\left[0, X_{e}\right]$.

Proof. Define the linear differential operator, $L[\cdot]$, as $L[w] \equiv w_{X X}+\frac{1}{2} X w_{X}-$ $\left(w-(1-p)^{1 /(1-p)}\right)$, for any suitably differentiable function $w(X)$. Now, $L\left[u_{l}\right]=$ $0 \forall X \in\left[0, X_{e}\right]$ and $u_{l}(0)=(1-p)^{1 /(1-p)}+\delta, u_{l X}(0)=-v \delta$. Also,

$$
L[\tilde{u}] \equiv \tilde{u}_{X X}+\frac{1}{2} X \tilde{u}_{X}-\left[\tilde{u}-(1-p)^{1 /(1-p)}\right]=-\left[\tilde{u}^{p}-\frac{1}{1-p} \tilde{u}\right]_{+}-\left[\tilde{u}-(1-p)^{1 /(1-p)}\right] \geqq 0
$$

$\forall-\infty<\tilde{u}<\infty$ and hence $\forall X \in\left[0, X_{e}\right]$. Moreover, $\tilde{u}(0)=u_{i}(0)$ and $\tilde{u}_{X}(0)=u_{l X}(0)$; thus we can apply the comparison theorem for initial value problems with linear ordinary 
differential operators (see, for example, Protter and Weinberger [23, Ch. 1, Theorem 13, p. 26]) to $\tilde{u}(X)$ and $u_{l}(X)$ in $\left[0, X_{e}\right]$ to obtain, $u_{l}(X) \leqq \tilde{u}(X)$ and $u_{l X}(X) \leqq$ $\tilde{u}_{x}(X) \forall X \in\left[0, X_{e}\right]$, as required.

We next consider a further initial value problem,

$$
\begin{gathered}
u_{X X}^{l}+\frac{1}{2} X u_{X}^{l}-N\left(u^{l}\right)_{+}=0, \quad X>0, \\
u^{l}(0)=(1-p)^{1 /(1-p)}+\delta, \quad u_{X}^{l}(0)=-v \delta
\end{gathered}
$$

where $N=1+\operatorname{Int}(1 / 1-p)$, and we shall henceforth refer to this initial value problem as LIVP2. The solution to LIVP2 is readily obtained. For $\nu \leqq 2^{2 N-1}(N !)^{2} /(2 N) ! \sqrt{\pi}$ then $u^{l}(X)$ is always positive in $X>0$ and,

$u^{l}(X)=(1-p)^{1 /(1-p)}+v \delta A_{2 N}(x) \int_{X}^{\infty} \frac{e^{-s^{2} / 4}}{A_{2 N}^{2}(s)} d s+\delta\left\{1-\frac{(2 N) ! \sqrt{\pi} v}{2^{2 N-1}(N !)^{2}}\right\} A_{2 N}(X), \quad X>0$

where $A_{2 N}(X)=\sum_{r=0}^{N} N ![(2 r) !(N-r) !]^{-1} X^{2 r}$. It is readily shown from (3.12) that in this case $u^{l}(X)>(1-p)^{1 /(1-p)} \forall X>0$. Now, for $v>2^{2 N-1}(N !)^{2} /(2 N) ! \sqrt{\pi}$, then there is a point $X=X^{*}>0$ at which $u^{l}\left(X^{*}\right)=0$, with $u^{l}(X)>0$ for $0 \leqq X<X^{*}$ and $u^{l}(X)<0$ for $X>X^{*}$. In this case $u^{l}(X)$ is given by (3.12) for $0 \leqq X \leqq X^{*}$, but has the form

$$
u^{l}(X)=u_{X}^{l}\left(X^{*}\right) \int_{X^{*}}^{X} e^{-\left(\left(s^{2}-X^{* 2}\right) / 4\right)} d s
$$

for $X>X^{*}$. We note that, in this case,

$$
u^{l}(X) \rightarrow u_{X}^{l}\left(X^{*}\right) \int_{X^{*}}^{\infty} e^{-\left(\left(s^{2}-X^{* 2}\right) / 4\right)} d s<0
$$

as $X \rightarrow \infty$. In addition we observe from $(3.12,13)$ that for any $v \geqq 0$, there is a constant $K(N)$ such that,

$$
u^{l}(X)<(1-p)^{1 /(1-p)}+\delta\left[1+K(N) X^{2 N}\right] \forall X \geqq 0
$$

We can now establish:

Proposition 3.16. Let $\tilde{u}(X)$ be a solution of IVP1 for $X \in\left[0, X_{e}\right]$ for any $X_{e}>0$. Then $\tilde{u}(X) \leqq u^{l}(X)$ and $\tilde{u}_{X}(X) \leqq u_{X}^{l}(X) \forall X \in\left[0, X_{e}\right]$.

Proof. We first observe that if $\tilde{u}(X) \ngtr 0 \forall X \in\left[0, X_{e}\right]$ then $\exists$ an $X_{0} \in\left[0, X_{e}\right]$ such that $\tilde{u}\left(X_{0}\right)=0, \tilde{u}(X)>0 \forall X \in\left[0, X_{0}\right]$ and $\tilde{u}(X) \equiv 0$ or $\tilde{u}(X)<0 \forall X \in\left(X_{0}, X_{e}\right]$. This follows from equation (3.1) and Proposition 2.5. There are now three cases to consider: 
(i) First suppose $u^{l}(X)>0 \forall X \in\left[0, X_{e}\right]$ and $\tilde{u}(X)>0 \forall X \in\left[0, X_{e}\right]$. We then define the linear differential operator, $L[\cdot]$, as $L[w] \equiv w_{X X}+\frac{1}{2} X w_{X}-N w$, for any suitably differential function $w(X)$. Now via LIVP2, $L\left[u^{l}\right]=0 \forall X \in\left[0, X_{e}\right]$. Also

$$
L[\tilde{u}] \equiv \tilde{u}_{X X}+\frac{1}{2} X \tilde{u}_{X}-N \tilde{u}=-\left[\tilde{u}^{p}-\frac{1}{1-p} \tilde{u}\right]-N \tilde{u} \leqq 0
$$

$\forall \tilde{u}>0$ and hence $\forall X \in\left[0, X_{e}\right]$. Moreover, $\tilde{u}(0)=u^{l}(0), \tilde{u}_{X}(0)=u_{X}^{l}(0)$, thus we can apply the comparison theorem for linear ordinary differential operators, [23], to $\tilde{u}(X)$ and $u^{l}(X)$ in $\left[0, X_{e}\right]$ to obtain $\tilde{u}(x) \leqq u^{l}(X)$ and $\tilde{u}_{X}(X) \leqq u_{X}^{l}(X) \forall X \in\left[0, X_{e}\right]$.

(ii) Next suppose $u^{l}(X)>0 \forall X \in\left[0, X_{e}\right]$, but $\tilde{u}(X) \ngtr 0 \forall X \in\left[0, X_{e}\right]$, and let $X_{0}$ be as defined above: For $X \in\left[0, X_{0}\right]$ the result follows from (i) above, whilst for $X \in\left[X_{0}, X_{e}\right]$ we apply the same argument as in (i) but using the operator $\bar{L}[w] \equiv w_{X X}+\frac{1}{2} X w$. For $X \in\left[X_{0}, X_{e}\right], \tilde{u}(X) \leqq 0$, from above. Thus, using equation (3.1), $\bar{L}[\tilde{u}]=0 \forall X \in\left[X_{0}, X_{e}\right]$. However, in this case $u^{l}(X)>0 \forall X \in\left[X_{0}, X_{e}\right]$ so that $\bar{L}\left[u^{l}\right]=N u^{l}>0 \forall X \in\left[X_{0}, X_{e}\right]$. Moreover, $u^{l}\left(X_{0}\right) \geqq \tilde{u}\left(X_{0}\right)$ and $u_{X}^{l}\left(X_{0}\right) \geqq \tilde{u}_{X}\left(X_{0}\right)$, and so the comparison theorem for linear differential operators, [12], gives $\tilde{u}(X) \leqq u^{l}(X)$ and $\tilde{u}_{X}(X) \leqq u_{X}^{l}(X) \forall X \in\left[X_{0}, X_{e}\right]$, as required.

(iii) Finally suppose $u^{l}(X) \ngtr 0$ on $\left[0, X_{e}\right]$. Then $\exists$ an $X=\bar{X}_{0}$ such that $u^{l}(X)>$ $0 \forall X \in\left[0, \bar{X}_{0}\right), u^{l}\left(\bar{X}_{0}\right)=0$ and $u^{l}(X)<0 \forall X \in\left[\bar{X}_{0}, X_{e}\right]$, via $(3.12,13)$. For $X \in\left[0, \bar{X}_{0}\right)$ the result follows from parts (i) and (ii). Moreover we can deduce that $\tilde{u}\left(\bar{X}_{0}\right) \leqq u^{l}\left(\bar{X}_{0}\right)=0$ and $\tilde{u}_{X}\left(\bar{X}_{0}\right) \leqq u_{X}^{l}\left(\bar{X}_{0}\right)<0$ from the result on $\left[0, \bar{X}_{0}\right)$ and continuity of $\tilde{u}(X), u^{l}(X)$ and first derivatives at $X=\bar{X}_{0}$. These conditions enable us to conclude that (via the first part of this proof and $(3.12,13)) \tilde{u}(X), u^{l}(X) \leqq 0 \forall X \in\left[\bar{X}_{0}, X_{e}\right]$, and so via (3.10), (3.1) $\bar{L}[\tilde{u}]=\vec{L}\left[u^{l}\right]=0 \forall X \in\left[\bar{X}_{0}, X_{e}\right]$, and the result follows via the comparison theorem.

All cases have now been considered and the proof is complete.

Remark 3.17. On the interval $X \in\left[0, X_{e}\right]$, for any $X_{e}>0$, Propositions 3.9, 3.16 show that, $u_{l}(X) \leqq \tilde{u}(X) \leqq u^{l}(X), u_{l X}(X) \leqq \tilde{u}_{X}(X) \leqq u_{X}^{l}(X)$, which provide $a$ priori bounds on the solution of IVP1.

Having established a priori bounds on the solution of IVP1, we are now able to consider (for each $\delta>0, v \geqq 0$ ) global existence and uniqueness of solutions to IVP1.

Proposition 3.18. For each $\delta>0$ and $0 \leqq v \leqq 1 / \sqrt{\pi}$ there exists a unique solution to IVP1 with $X \in\left[0, X_{e}\right]$, for any $X_{e}>0$.

Proof. We first write IVP1 as the equivalent first order system

$$
\left.\begin{array}{l}
\tilde{u}_{X}=\tilde{V}, \quad \tilde{V}_{X}=-\frac{1}{2} X \tilde{V}-\left[\tilde{u}^{p}-\frac{1}{1-p} \tilde{u}\right]_{+}, \quad X \in\left[0, X_{e}\right] \\
\tilde{u}(0)=(1-p)^{1 /(1-p)}+\delta, \quad \tilde{V}(0)=-v \delta .
\end{array}\right\}
$$


Now, via Propositions 3.9, 3.16 and 3.17 , any solution of (3.19) is a priori bounded in $\left[0, X_{e}\right]$ with, for $0 \leqq v \leqq 1 / \sqrt{\pi}$,

$$
(1-p)^{1 /(1-p)} \leqq \tilde{u}(X) \leqq(1-p)^{1 /(1-p)}+\delta\left[1+K(N) X_{e}^{2 N}\right], \quad-\frac{\delta}{\sqrt{\pi}} \leqq \tilde{V}(X) \leqq 2 N \delta K(N) X_{e}^{2 N-1}
$$

Now let $D=\hat{R} \times\left[0, X_{e}\right]$, where $\hat{R}$ is the rectangle described in (3.20), and define $F: D \rightarrow \mathbb{R}^{2}$ as,

$$
F(\tilde{u}, \tilde{V}, X)=\left(\tilde{V},-\frac{1}{2} X \tilde{V}-\left[\tilde{u}^{p}-\frac{1}{1-p} \tilde{u}\right]_{+}\right)
$$

It is clear that $F$ is continuous throughout $D$. Moreover, since via (3.20) $\tilde{u}$ is bounded away from zero in $D$, then $F$ is a differentiable function of $(\tilde{u}, \tilde{V})$ throughout $D$, and hence is Lipschitz continuous in $(\tilde{u}, \tilde{V})$ throughout $D$. Under these conditions, a repeated application of the local existence and uniqueness theorem (see, for example, Coddington and Levinson [8, Ch. 1., Theorem 2.3]) on the intervals $[0, \alpha],[\alpha, 2 \alpha], \ldots,[(s-1) \alpha, s \alpha]$ (where $\alpha=\min \left(X_{e}, b / M\right) \quad$ with, $\quad b=\frac{1}{2}(1-p)^{1 /(1-p)}+1, \quad M=\max |F(\tilde{u}, \tilde{V}, X)| \forall(\tilde{u}, \tilde{v}, X) \in$ $\left[\frac{1}{2}(1-p)^{1 /(1-p)}, \frac{3}{2}(1-p)^{1 /(1-p)}+\delta\left(1+K(N) X_{e}^{2 N}\right)\right] \times\left[-(1+\delta / \sqrt{\pi}), 2 N \delta K(N) X_{e}^{2 N-1}+1\right] \times$ $\left[0, X_{e}\right]$, and $s \in \mathbb{N}$ with $\left.X_{e} / \alpha \leqq s<X_{e} / \alpha+1\right)$ establishes existence and uniqueness on the interval $X \in\left[0, X_{e}\right]$, for any $X_{e}>0$.

Remark 3.19. For the above proof, in the notation of Coddington and Levinson [8], the rectangle $R$ used in each local application of [8, Theorem 2.3], with initial conditions $\left(\tilde{u}_{0}, \tilde{v}_{0}\right)$ at $X_{0}$, is $\left|\tilde{u}-\tilde{u}_{0}\right| \leqq \frac{1}{2}(1-p)^{1 /(1-p)},\left|\tilde{v}-\tilde{v}_{0}\right| \leqq 1,\left|X-X_{0}\right| \leqq 1$.

The restriction $0 \leqq v \leqq 1 / \sqrt{\pi}$ in Proposition 3.18 can be removed as follows:

Extension 3.20. For $v>1 / \sqrt{\pi}$ existence can again be established on $\left[0, X_{e}\right]$, for any $X_{e}>0$, via the a priori bounds of Propositions 3.9, 3.16 and the Cauchy-Peano local existence theorem ([8, Ch. 1, Theorem 1.2]). However, in this case the lower bound on $\tilde{u}$ is negative, and so uniqueness cannot be guaranteed immediately as now $F$ is not Lipschitz continuous in $(\tilde{u}, \tilde{v})$ throughout $D(D$ now contains part of the plane $\tilde{u}=0)$. Despite this, uniqueness can still be established.

Proof (of uniqueness for $v>1 / \sqrt{\pi}$ ). Suppose $\tilde{u}(X ; v)$ is a solution of IVP1 with $v>1 / \sqrt{\pi}$ and $X \in\left[0, X_{e}\right]$. There are two cases to consider,

(i) $\tilde{u}(X ; v)>0 \forall X \in\left[0, X_{e}\right]$

Uniqueness follows from applying the local uniqueness result $([8, \mathrm{Ch}$. 1 , Theorem 2.3]) at each $X_{0} \in\left[0, X_{e}\right]$, as $F(\tilde{u}, \tilde{V}, X)$ is locally Lipschitz continuous at each such point $\left(\tilde{u}\left(X_{0}\right), \tilde{V}\left(X_{0}\right), X_{0}\right)$, since $\tilde{u}(X ; v)$ is bounded away from zero.

(ii) $\tilde{u}(X ; v) \ngtr 0 \forall X \in\left[0, X_{e}\right]$ 
In this case $\exists X^{*} \in\left(0, X_{e}\right]$ such that $\tilde{u}\left(X^{*} ; v\right)=0$ and $\tilde{u}(X, v)>0 \forall X \in\left[0, X^{*}\right)$. Uniqueness for $X \in\left[0, X^{*}\right)$ follows as in (i) above. At $X=X^{*}, \tilde{u}_{X}\left(X^{*} ; v\right) \leqq 0$. With $\tilde{u}_{X}\left(X^{*} ; v\right)=0$, then via equation (3.1) and Proposition 2.5, we deduce that $\tilde{u}(X ; y) \equiv 0$ for $X \in\left[X^{*}, X_{e}\right]$, and uniqueness follows on this interval. The remaining possibility is that $\tilde{u}_{X}\left(X^{*} ; v\right)<0$, when for $X \in\left[X^{*}, X_{e}\right], \tilde{u}(X ; v)$ satisfies the initial value problem (via IVP1),

$$
\begin{aligned}
& \tilde{u}_{X X}+\frac{1}{2} X \tilde{u}_{X}=0, \quad X \in\left[X^{*}, X_{e}\right] \\
& \tilde{u}\left(X^{*} ; v\right)=0, \quad \tilde{u}_{X}\left(X^{*} ; v\right)=-\alpha^{*}
\end{aligned}
$$

for some $\alpha^{*}>0$. This has the unique solution

$$
\tilde{u}(X ; v)=-\alpha^{*} \int_{X^{*}}^{X} e^{-\left(\left(s^{2}-X^{* 2}\right) / 4\right)} d s<0
$$

$\forall X \in\left[X^{*}, X_{e}\right]$, and the result is established.

The next stage is to examine the closeness of solutions to IVP1 and LIVP1. We begin with:

Lemma 3.21. Let $\tilde{u}(X)$ and $u_{l}(X)$ be solutions of IVP1 and LIVP1 respectively on $\left[0, X_{e}\right]$, for any $X_{e}>0$, then,

(i) $0 \leqq H\left(u_{l}\right)-H(\tilde{u}) \leqq \frac{1}{1-p}\left(\tilde{u}-u_{l}\right), \quad 0 \leqq v \leqq 1 / \sqrt{\pi}$,

(ii) $0 \leqq H_{l}\left(u_{l}\right)-H\left(u_{l}\right) \leqq \Lambda(p) \delta^{2}, \quad v=1 / \sqrt{\pi}$,

for all $X \in\left[0, X_{e}\right]$. Here $H(w)=\left[w^{p}-(1 /(1-p)) w\right]+$ and $H_{l}(w)=-\left[w-(1-p)^{1 /(1-p)}\right]$, with $\Lambda(p)=\frac{1}{2} p(1-p)^{-1 /(1-p)}$.

Proof. (i) Via Proposition 3.9 we have $\tilde{u}(X) \geqq u_{l}(X) \forall X \in\left[0, X_{e}\right]$. Moreover $u_{l}(X)>$ $(1-p)^{1 /(1-p)} \forall X>0$. Hence $\tilde{u}(X) \geqq(1-p)^{1 /(1-p)} \forall X \in\left[0, X_{e}\right]$. Now, $H(w)$ is strictly monotone decreasing in $w$ for $w>(1-p)^{1 /(1-p)}$. Therefore $\left[H\left(u_{l}(X)\right)-H(\tilde{u}(X))\right] \geqq$ $0 \forall X \in\left[0, X_{e}\right]$. In addition, $H(w)$ is also Lipschitz continuous in $w>(1-p)^{1 /(1-p)}$ (it is differentiable, with bounded derivative $\left.\left|H^{\prime}(w)\right| \leqq(1 / 1-p) \forall w \geqq(1-p)^{1 /(1-p)}\right)$. Thus $\left[H\left(u_{l}(X)\right)-H(\tilde{u}(X))\right] \leqq(1 / 1-p)\left[\tilde{u}(X)-u_{l}(X)\right] \forall X \in\left[0, X_{e}\right]$, as required.

(ii) We note first that when $v=1 / \sqrt{\pi}, u_{l}(X)$ is monotone decreasing in $X \geqq 0$, with $u_{l}(X) \rightarrow(1-p)^{1 /(1-p)}$ as $X \rightarrow \infty$. Also, in $w \geqq(1-p)^{1 /(1-p)}, H_{l}(w)-H(w)$ is positive and monotone increasing. Therefore $0 \leqq H_{l}\left(u_{l}(X)\right)-H\left(u_{l}(X)\right) \leqq H_{l}\left(u_{l}(0)\right)-H\left(u_{l}(0)\right) \leqq \Lambda(p) \delta^{2} \forall$ $X \in\left[0, X_{e}\right]$, on using $u_{l}(0)=(1-p)^{1 /(1-p)}+\delta$ and Taylor's theorem with remainder.

Extension 3.22. The inequality (i) also holds for $v>1 / \sqrt{\pi}$, but only extends to the maximal interval $\left[0, \hat{X}_{0}\right]$, where $\hat{X}_{0}$ is the unique, positive value of $X$ with $u_{1}\left(\hat{X}_{0}\right)=$ $\left.[p(1-p)]^{1 /(1-p)}<(1-p)\right]^{1 /(1-p)}$. Note that $\hat{X}_{0}$ depends on $\delta$ and $v$.

The inequality (ii) holds for $v>1 / \sqrt{\pi}$, but only extends to the maximal interval $\left[0, \hat{X}_{1}\right]$, where $\hat{X}_{1}$ is the unique, positive value of $X$ with $u_{l}\left(\hat{X}_{1}\right)=\max \left\{(1-p)^{1 /(1-p)}-\delta\right.$, 
$\left.[p(1-p)]^{1 /(1-p)}\right\}$. This inequality also holds for $0 \leqq v<1 / \sqrt{\pi}$, but only extends to the maximal interval $\left[0, \hat{X}_{2}\right]$, where $\hat{X}_{2}$ is the unique, positive value of $X$ with $u_{1}\left(\hat{X}_{2}\right)=$ $(1-p)^{1 /(1-p)}+\delta$. Again we note that both $\hat{X}_{1}$ and $\hat{X}_{2}$ will depend on $\delta$ and $v$.

We next write IVP1 and LIVP1 as equivalent first order systems,

$$
\left.\begin{array}{ll}
\tilde{u}_{X}=\tilde{v}, \tilde{v}_{X}=-\frac{1}{2} X \tilde{v}-H(\tilde{u}) \quad ; & X>0 \\
u_{l X}=v_{l}, v_{l X}=-\frac{1}{2} X v_{l}-H_{l}\left(u_{l}\right) ; & X>0
\end{array}\right\}
$$

subject to $u_{l}(0)=\tilde{u}(0)=(1-p)^{1 /(1-p)}+\delta, \quad v_{l}(0)=\tilde{v}(0)=-v \delta$. On defining $W(X)=$ $\left(\tilde{u}(X)-u_{l}(X), \tilde{v}(X)-v_{l}(X)\right)^{T}$, we readily find from (3.23) that $W(X)$ satisfies the following initial value problem

$$
W_{X}=A(X) W+g(W), \quad W(0)=0, \quad X>0,
$$

where

$$
A(X)=\left(\begin{array}{cc}
0 & 1 \\
0 & -\frac{1}{2} X
\end{array}\right), \quad g(W)=\left(0, H_{l}\left(u_{l}\right)-H(\tilde{u})\right)^{T}
$$

The initial value problem (3.24) is equivalent to the integral equation,

$$
W(X)=\int_{s=0}^{s=X} B(X) B^{-1}(s) g(W(s)) d s, \quad X>0,
$$

where $B(X)$ is a fundamental matrix for the system $Y_{X}=A(X) Y$, and can be taken as,

$$
B(X)=\left(\begin{array}{cc}
1 & \sqrt{\pi} \operatorname{erfc}\left(\frac{1}{2} X\right) \\
0 & -e^{-X^{2} / 4}
\end{array}\right)
$$

On substitution into (3.26) using (3.25) and (3.27) we arrive at,

$$
W(X)=\int_{s=0}^{s=X}\left[\sqrt{\pi}\left[\operatorname{erf}\left(\frac{1}{2} X\right)-\operatorname{erf}\left(\frac{1}{2} s\right)\right], e^{-\left(1 / 4 X^{2}\right)}\right]^{T} e^{\left(1 / 4 s^{2}\right)} \times\left[H_{l}\left(u_{l}(s)\right)-H(\tilde{u}(s))\right] d s, \quad X>0,
$$

which leads directly to the inequality,

$$
\begin{aligned}
|W(X)| \leqq & \int_{s=0}^{s=X}\left\{\sqrt{\pi}\left[\operatorname{erf}\left(\frac{1}{2} X\right)-\operatorname{erf}\left(\frac{1}{2} s\right)\right]+e^{-\left(1 / 4 X^{2}\right)}\right\} e^{\left(1 / 4 s^{2}\right)} \\
& \times\left|H_{l}\left(u_{l}(s)\right)-H(\tilde{u}(s))\right| d s, \quad X>0
\end{aligned}
$$

We can now establish: 
Proposition 3.29. Let $\tilde{u}(X)$ and $u_{l}(X)$ be solutions of IVP1 and LIVPi respectively. Then for any $\delta>0, v \geqq 0$,

$$
\left.\begin{array}{l}
\left|\tilde{u}(X)-u_{l}(X)\right| \\
\left|\tilde{u}_{X}(x)-u_{l X}(X)\right|
\end{array}\right\} \leqq \frac{1}{2} \Lambda(p) \delta^{2} X_{e}\left(X_{e}+2\right) \exp \left\{\frac{\left(X_{e}+1\right)}{(1-p)} X\right\}
$$

for all $X \in\left[0, X_{e}\right]$ (where $X_{e}$, when necessary, is restricted to those values allowable for Lemma 3.21 to hold).

Proof. From (3.28) we have immediately that

$$
|W(X)| \leqq \int_{s=0}^{s=X}[(X-s)+1]\left|H_{l}\left(u_{l}(s)\right)-H(\tilde{u}(s))\right| d s, \quad X>0 .
$$

Now, for $X \in\left[0, X_{e}\right]$ (with $X_{e}$, if necessary, restricted so that Lemma 3.21 holds) we have, via Lemma 3.21 and (3.22),

$$
\begin{aligned}
0 \leqq H_{l}\left(u_{l}\right)-H(\tilde{u}) & =\left[H_{l}\left(u_{l}\right)-H\left(u_{l}\right)\right]+\left[H\left(u_{l}\right)-H(\tilde{u})\right] \\
& \leqq \frac{1}{(1-p)}(\tilde{u}-u)+\Lambda(p) \delta^{2}
\end{aligned}
$$

$\forall s \in[0, X] \subseteq\left[0, X_{e}\right]$. Thus, using (3.31a) in (3.30) we arrive at,

$$
|W(X)| \leqq \int_{s=0}^{s=x}[(X-s)+1]\left\{\frac{1}{(1-p)}|W(s)|+\Lambda(p) \delta^{2}\right\} d s
$$

$\forall X \in\left[0, X_{e}\right]$. This leads to,

$$
|W(X)| \leqq \frac{\left(X_{e}+1\right)}{(1-p)} \int_{s=0}^{s=X}|W(s)| d s+\Lambda(p) \delta^{2}\left(\frac{1}{2} X_{e}^{2}+X_{e}\right),
$$

$\forall X \in\left[0, X_{e}\right]$. It is now straighforward to apply the Gronwall inequality (see for example, Hirsch and Smale $[13, \mathrm{Ch} .8, \S 4])$ to $(3.31 \mathrm{~b})$, to obtain,

$$
|W(X)| \leqq \frac{1}{2} \Lambda(p) \delta^{2} X_{e}\left(X_{e}+2\right) \exp \left\{\frac{\left(X_{e}+1\right)}{(1-p)} X\right\}
$$

$\forall X \in\left[0, X_{e}\right]$, as required.

Remark 3.32. For any finite (allowable) $X_{e}$, Proposition 3.29 implies that $\mid \tilde{u}(X)-$ $u_{l}(X)|,| \tilde{u}_{X}(X)-u_{l X}(X) \mid=0\left(\delta^{2}\right)$ uniformly on $X \in\left[0, X_{e}\right]$ as $\delta \rightarrow 0^{+}$for fixed $v \geqq 0$. 
We next make use of Proposition 3.29 to examine the behaviour of the solution to IVP1 with varying $v \geqq 0$, at a fixed $\delta \geqq 0$. We first recall that, for $v>1 / \sqrt{\pi}$, then $u_{l}(X)$ is monotone decreasing in $X$ with $u=0$ at $X=X_{c}(v, \delta)$ where, from (3.7), (3.8),

$$
\begin{aligned}
& \frac{2(1-p)^{1 /(1-p)}}{\delta \sqrt{\pi(v-1 / \sqrt{\pi})}} \quad \text { as } \quad v \rightarrow \frac{1^{+}}{\sqrt{\pi}} \\
& X_{c}(v, \delta) \sim \\
& \frac{(1-p)]^{1 /(1-p)}}{\delta v} \quad \text { as } \quad v \rightarrow \infty .
\end{aligned}
$$

Recall also that for $v>1 / \sqrt{\pi}, \hat{X}_{1}(v, \delta)$ is defined so that $u_{l}\left(\hat{X}_{1}\right)=\operatorname{Max}\left\{(1-p)^{1 /(1-p)}-\delta\right.$, $\left.(p(1-p))^{1 /(1-p)}\right\}$, and in this case Proposition 3.29 applies for $X \in\left[0, \hat{X}_{1}(v, \delta)\right]$. Hence, applying Proposition 3.29 at $X=\hat{X}_{1}(v, \delta)$ we obtain

$$
\begin{aligned}
\tilde{u}\left(\hat{X}_{1}(v, \delta)\right)-(1-p)^{1 /(1-p)} & \leqq \max \left\{-\delta,-(1-p)^{1 /(1-p)}\left(1-p^{1 /(1-p)}\right)\right\} \\
& +\frac{1}{2} \Lambda(p) \delta^{2} \hat{X}_{1}\left(\hat{X}_{1}+2\right) \exp \left\{\frac{\left(\hat{X}_{1}+1\right) \hat{X}_{1}}{(1-p)}\right\}
\end{aligned}
$$

We also note, via (3.7), that,

$$
\hat{X}_{1}(v, \delta) \rightarrow\left\{\begin{array}{lll}
\infty & \text { as } & v \rightarrow 1^{+} / \sqrt{\pi} \\
0 & \text { as } & v \rightarrow \infty
\end{array}\right.
$$

with $\hat{X}_{1}(v, \delta)$ being a monotone decreasing function of $v>1 / \sqrt{\pi}$. Therefore, for a fixed $\delta>0$, we observe from (3.34), (3.35) that there exists a $v=v_{u}(\delta)>1 / \sqrt{\pi}$ (with $v_{u}(\delta) \rightarrow 1^{+} / \sqrt{\pi}$ as $\delta \rightarrow 0^{+}$) such that,

$$
\tilde{u}\left(\hat{X}_{1}(v, \delta)\right)<(1-p)^{1 /(1-p)} \forall v \in\left(v_{u}(\delta), \infty\right)
$$

Moreover (via equation (3.1), the only turning point of $\tilde{u}$ with $0<\tilde{u}<(1-p)^{1 /(1-p)}$ can be a local maximum) we may also infer that,

$$
\tilde{u}_{X}\left(\hat{X}_{1}(v, \delta)\right)<0 \forall v \in\left(v_{u}(\delta), \infty\right) \text {. }
$$

Thus, using $(3.36,37)$ and equation $(3.1)$, it is clear that for each $v \in\left(v_{u}(\delta), \infty\right)$, then $\tilde{u}(X)$ is monotone decreasing for $0<X<X^{*}(v)\left(X^{*}(v)>X_{c}(v)\right)$ with $\tilde{u}\left(X^{*}(v)\right)=0$. For $X>$ $X^{*}(v)$ we have (from (3.1) directly),

$$
\tilde{u}(X)=u_{X}\left(X^{*}(v)\right) \int_{X^{*}(v)}^{X} e^{-\left(s^{2}-X^{* 2}\right) / 4} d s,
$$

with, 


$$
\tilde{u}(X) \rightarrow u_{X}\left(X^{*}(v)\right) \int_{X^{*}(v)}^{\infty} e^{-\left(s^{2}-X^{* 2}\right) / 4} d s \leqq 0
$$

as $X \rightarrow \infty$.

We now consider the case when $0 \leqq v<1 / \sqrt{\pi}$. In this case recall that $u_{l}(X)>$ $(1-p)^{1 /(1-p)}$ in $X>0$ and has a single turning point, which is a local minimum, with $u_{1}(X) \rightarrow \infty$ as $X \rightarrow \infty$. Moreover, there exists a unique point $X=\hat{X}_{2}(v, \delta)$ with $u_{l}\left(\hat{X}_{2}\right)=$ $(1-p)^{1 /(1-p)}+\delta$, and Proposition 3.29 holds for $X \in\left[0, \hat{X}_{2}\right]$. We also observe that (via (3.7)),

$$
\hat{X}_{2}(v, \delta) \rightarrow\left\{\begin{array}{lll}
\infty & \text { as } & v \rightarrow 1^{-} / \sqrt{\pi} \\
0 & \text { as } & v \rightarrow 0^{+}
\end{array}\right.
$$

Thus, for fixed $\delta>0, \tilde{u}\left(\hat{X}_{2}\right)>u_{l}\left(\hat{X}_{2}\right)>(1-p)^{1 /(1-p)}$ and $\tilde{u}_{X}\left(\hat{X}_{2}\right)>u_{l X}\left(\hat{X}_{2}\right)>0$, via Proposition 3.9 , for all $v \in[0,1 / \sqrt{\pi}$ ). These conditions imply (using (3.1)) that $\tilde{u}(X)$ is monotone increasing in $X>\hat{X}_{2}(v, \delta)$ with $\tilde{u}(X) \rightarrow \infty$ as $X \rightarrow \infty, \forall v \in[0,1 / \sqrt{\pi})$. We have thus established:

Lemma 3.39. For any $\delta>0$, then,

(i) with $v \in\left(v_{u}(\delta), \infty\right), \tilde{u}(X)$ is monotone decreasing with $\tilde{u}(X) \rightarrow \tilde{u}_{\infty} \leqq 0$ as $X \rightarrow \infty$. Here $v_{u}(\delta)>1 / \sqrt{\pi} \forall \delta>0$, with $v_{u}(\delta) \rightarrow 1^{+} / \sqrt{\pi}$ as $\delta \rightarrow 0^{+}$.

(ii) with $v \in[0,1 / \sqrt{\pi}), \tilde{u}(X)>(1-p)^{1 /(1-p)} \forall X>0$. Moreover, $\tilde{u}(X)$ is monotone increasing in $X>\hat{X}_{2}(v)$, and $\tilde{u}(X) \rightarrow \infty$ as $X \rightarrow \infty$.

In what follows we regard $\delta>0$ as fixed and write $\tilde{u}(X)=\tilde{u}(X, v)$ as we wish to explore the dependence of $\tilde{u}$ on the parameter $v \geqq 0$.

Lemma 3.40. Let $I_{\delta}=\left\{v \in \mathbb{R}^{+} \cup\{0\}: \tilde{u}(X, v) \geqq(1-p)^{1 /(1-p)} \forall X \geqq 0\right\}$, then $I_{\delta}=\left[0, v^{*}(\delta)\right]$ for some $1 / \sqrt{\pi} \leqq v^{*}(\delta) \leqq v_{u}(\delta)$.

Proof. We have already shown that $[0,1 / \sqrt{\pi}) \subseteq I_{\delta}$. Thus $\inf \left(I_{\delta}\right)=0$ and putting $\sup \left(I_{\delta}\right)=v^{*}(\delta)$, then, $1 / \sqrt{\pi} \leqq v^{*}(\delta) \leqq v_{u}(\delta)$. We now show that $I_{\delta}$ is connected. Suppose that $v_{1} \in I_{\delta}\left(v_{1}>0\right)$, then $\tilde{u}_{1}(X) \equiv \tilde{u}\left(x, v_{1}\right) \geqq(1-p)^{1 /(1-p)} \forall X \geqq 0$. Also let $0<v_{0}<v_{1}$ with $\tilde{u}_{0}(X) \equiv \tilde{u}\left(X, v_{0}\right)$. From equation (3.1),

$$
\tilde{u}_{0}^{\prime \prime}+\frac{1}{2} X \tilde{u}_{0}^{\prime}+\left(\tilde{u}_{0}^{p}-\frac{1}{1-p} \tilde{u}_{0}\right)_{+}=\tilde{u}_{1}^{\prime \prime}+\frac{1}{2} X \tilde{u}_{1}^{\prime}+\left(\tilde{u}_{1}^{p}-\frac{1}{1-p} \tilde{u}_{1}\right)_{+}=0
$$

$\forall X \geqq 0$, and $\tilde{u}_{0}(0)=\tilde{u}_{1}(0), \tilde{u}_{0}^{\prime}(0)>\tilde{u}_{1}^{\prime}(0)$. Thus, via the nonlinear comparison theorem for ordinary differential operators (Protter and Weinberger [23, Ch. 1, §9, Theorem 23]) we have $\tilde{u}_{0}(X) \geqq \tilde{u}_{1}(X) \forall X \geqq 0$ and therefore $v_{0} \in I_{\delta}$. We conclude that $I_{\delta}$ is connected. Finally, we must show that $v^{*}(\delta) \in I_{\delta}$ (and hence that $I(\delta)$ is closed). If we suppose that 
$v^{*}(\delta) \notin I_{\delta}$, then $\exists$ an $\bar{X}>0$ such that $\tilde{u}\left(\bar{X}, v^{*}\right)<(1-p)^{1 /(1-p)}$. However, $\tilde{u}(\bar{X}, v) \geqq$ $(1-p)^{1 /(1-p)} \forall 0 \leqq v<v^{*}(\delta)$, and so $\tilde{u}(\bar{X}, v)$ cannot be continuous in $v$ at $v=v^{*}(\delta)$. This contradicts continuous dependence at $X=\bar{X}$ of the solution of IVP1 on initial conditions (Coddington and Levinson [8, Ch. 1, $\S$, Theorem 7.1]), and hence $v^{*}(\delta) \in I_{\delta}$. The result follows.

For each $\delta>0$, we now consider the solution of IVP1 with $v=v^{*}(\delta)$.

Lemma 3.41. The solution $\tilde{u}\left(X, v^{*}\right)$ of IVP1 (for any $\left.\delta>0\right)$ is monotone decreasing in $X \geqq 0$ and has $\tilde{u}\left(X, v^{*}\right) \rightarrow(1-p)^{1 /(1-p)}$ as $X \rightarrow \infty$.

Proof. Suppose $\tilde{u}\left(X, v^{*}\right) \rightarrow \infty$ as $X \rightarrow \infty$, then $\exists$ an $X^{*}>0$ such that $\tilde{u}\left(X^{*}, v^{*}\right)>1+$ $\delta+(1-p)^{1 /(1-p)}$. However $\forall v>v^{*}(\delta), \tilde{u}\left(X^{*}, v\right)<\delta+(1-p)^{1 /(1-p)}$. This contradicts continuity of $\tilde{u}\left(X^{*}, v\right)$ on $v$ at $v=v^{*}(\delta)$. Therefore we conclude that $\tilde{u}\left(X, v^{*}\right)$ must remain bounded as $X \rightarrow \infty$. Since $\tilde{u}\left(X, v^{*}\right) \geqq(1-p)^{1 /(1-p)}$, then (via $\left.(3.1)\right) \tilde{u}\left(X, v^{*}\right)$ can have at most one turning point in $X>0$, which must be a local minimum. We suppose that $\tilde{u}\left(X, v^{*}\right)$ has a local minimum at $X=X_{m}>0$ with $\tilde{u}\left(X_{m}, v^{*}\right) \geqq(1-p)^{1 /(1-p)}$. Then $\tilde{u}\left(X, v^{*}\right)$ is monotone increasing and bounded above in $X>X_{m}$, so $\tilde{u}\left(X, v^{*}\right) \rightarrow u_{\infty}$ as $X \rightarrow \infty$, with $u_{\infty}>(1-p)^{1 /(1-p)}$. However, this is not compatible with equation (3.1), and we conclude that $\tilde{u}\left(X, v^{*}\right)$ is monotone decreasing in $X \geqq 0$. Thus $\tilde{u}\left(X, v^{*}\right) \rightarrow u_{\infty}$ as $X \rightarrow \infty$ with now $(1-p)^{1 /(1-p)} \leqq u_{\infty}<(1-p)^{1 /(1-p)}+\delta$. Equation (3.1) then gives immediately $u_{\infty}=$ $(1-p)^{1 /(1-p)}$, as required.

Remark 3.42. It follows from Lemmas 3.40, 3.41 that for all $v \in\left(v^{*}(\delta), \infty\right)$, then $\tilde{u}(X, v)$ is monotone decreasing in $X$, with $\tilde{u}(X, v) \rightarrow \tilde{u}_{\infty} \leqq 0$ as $X \rightarrow \infty$. Note also that $v^{*}(\delta) \rightarrow 1^{+} / \sqrt{\pi}$ as $\delta \rightarrow 0^{+}$(via Lemma 3.40 ).

At present we have shown that for any $\delta>0$, there is at least one value of $v$, given by $v=v^{*}(\delta)$, such that the solution of IVP1 is asymptotic to $(1-p)^{1 /(1-p)}$ as $X \rightarrow \infty$. We now determine that $v=v^{*}(\delta)$ is the only value of $v$ for which the solution of IVP1 has this property.

Lemma 3.43. Let $J_{\delta}=\left\{v \in \mathbb{R}^{+} \cup\{0\}: \tilde{u}(X, v) \rightarrow(1-p)^{1 /(1-p)}\right.$ as $\left.X \rightarrow \infty\right\}$, then $J_{\delta}=$ $\left[v_{*}(\delta), v^{*}(\delta)\right]$ for some $1 / \sqrt{\pi} \leqq v_{*}(\delta) \leqq v^{*}(\delta)$.

Proof. From Remark 3.42 we have immediately that $J_{\delta} \subseteq I_{\delta}$, and, via Lemma 3.41, $\sup \left(J_{\delta}\right)=v^{*}(\delta) \in J_{\delta}$. Let $v_{*}(\delta)=\inf \left(J_{\delta}\right)$, then $v_{*}(\delta) \geqq 1 / \sqrt{\pi}$ via Lemma 3.39. To demonstrate that $J_{\delta}$ is connected, we follow the proof of Lemma 3.40 and use the nonlinear comparison theorem for ordinary differential operators, [23]. Finally to show that $v_{*}(\delta) \in J_{\delta}$ we again follow the proof of Lemma 3.40, using continuous dependence of the solution of IVP1 on $v$ at fixed $X,[8]$.

We note from Lemma 3.43 and Remark 3.42 that $\nu_{*}(\delta) \rightarrow 1^{+} / \sqrt{\pi}$ as $\delta \rightarrow 0^{+}$. Moreover we are able to show that for each $\delta>0, J_{\delta}$ has just one element. 
Lemma 3.44. For each $\delta>0$, we have $v^{*}(\delta)=v_{*}(\delta)$.

Proof. Suppose $\exists$ a $\delta>0$ such that $v^{*}(\delta) \neq v_{*}(\delta)$. Then, by definition $v_{*}(\delta)<v^{*}(\delta)$ and $\exists$ values $v=v_{0}, v_{1}$ with $v_{*}(\delta)<v_{0}<v_{1}<v^{*}(\delta)$. Let $\tilde{u}_{1}(X)=\tilde{u}\left(X, v_{1}\right)$ and $\tilde{u}_{0}(X)=\tilde{u}\left(X, v_{0}\right)$, then, via the nonlinear comparison theorem, [23], it is readily deduced that,

$$
\psi(X) \geqq 0 \forall X \geqq 0,
$$

where $\quad \psi(X) \equiv \tilde{u}_{0}(X)-\tilde{u}_{1}(X)$ in $X \geqq 0$. Moreover, using initial conditions $\left(\tilde{u}_{0}(0)=\tilde{u}_{1}(0)=(1-p)^{1 /(1-p)}+\delta\right)$ and since $v_{0}, v_{1} \in J_{\delta}$, then,

$$
\psi(0)=0, \quad \psi(X) \rightarrow 0^{+} \quad \text { as } \quad X \rightarrow \infty .
$$

Also, $\psi^{\prime}(0)=-\delta\left(v_{0}-v_{1}\right)>0$ and so $\exists$ an $X_{+}>0$ such that,

$$
\psi(X)>0 \forall X \in\left(0, X_{+}\right)
$$

The conditions $(3.45-47)$ imply that $\exists$ a point $X=X^{T}>0$. where $\psi(X)$ has a local maximum. Thus,

$$
\psi\left(X^{T}\right)>0, \quad \psi^{\prime}\left(X^{T}\right)=0, \quad \psi^{\prime \prime}\left(X^{T}\right) \leqq 0
$$

Now as both $\tilde{u}_{1}(X)$ and $\tilde{u}_{0}(X)$ are solutions of IVP1 with $v=v_{1}, v_{0}$ respectively, then $\psi(X)$ satisfies the following,

$$
\psi^{\prime \prime}+\frac{1}{2} X \psi^{\prime}=\frac{1}{(1-p)}\left[\tilde{u}_{0}(X)-\tilde{u}_{1}(X)\right]-\left[\tilde{u}_{0}^{p}(X)-\tilde{u}_{1}^{p}(X)\right]
$$

in $X>0$. We now consider $X=X^{T}$. Since $\psi\left(X^{T}\right)>0$, then, $\tilde{u}_{0}\left(X^{T}\right)>\tilde{u}_{1}\left(X^{T}\right)>(1-p)^{1 /(1-p)}$ (via definition of $J_{\delta}$ ). Thus, using the mean value theorem,

$$
\tilde{u}_{0}^{p}\left(X^{T}\right)-\tilde{u}_{1}^{p}(X)=p \xi^{p-1}\left[\tilde{u}_{o}\left(X^{T}\right)-\tilde{u}_{1}\left(X^{T}\right)\right],
$$

where $\xi \in\left(\tilde{u}_{1}, \tilde{u}_{0}\right)$. Hence,

$$
\begin{aligned}
\tilde{u}_{0}^{p}\left(X^{T}\right)-\tilde{u}_{1}^{p}\left(X^{T}\right) & <\frac{p}{(1-p)}\left[\tilde{u}_{0}\left(X^{T}\right)-\tilde{u}_{1}\left(X^{T}\right)\right] \\
& <\frac{p}{(1-p)}\left[\tilde{u}_{0}\left(X^{T}\right)-\tilde{u}_{1}\left(X^{T}\right)\right]
\end{aligned}
$$

Next, evaluating (3.49) at $X=X^{T}$, using (3.50), we arrive at, $\psi^{\prime \prime}\left(X^{T}\right)=(1 /(1-p))$ $\left\{\tilde{u}_{0}\left(X^{T}\right)-\tilde{u}_{1}\left(X^{T}\right)\right\}-\left\{\tilde{u}_{0}^{p}\left(X^{T}\right)-\tilde{u}_{1}^{p}\left(X^{T}\right)\right\}>0$, which contradicts the last of (3.48). We conclude that $v^{*}(\delta) \equiv \nu_{*}(\delta) \forall \delta>0$, as required. 
In the light of the above lemma we introduce the notation $v_{c}(\delta)=v_{*}(\delta)=v^{*}(\delta)$. We can now state:

Theorem 3.51. For each $\delta>0$ the solution of IVP1 is such that $\tilde{u}(X, v) \rightarrow(1-p)^{1 /(1-p)}$ as $X \rightarrow \infty$ if and only if $v=v_{c}(\delta)$. Moreover, $\tilde{u}\left(X, v_{c}(\delta)\right)$ is monotone decreasing in $X \geqq 0$, and $v_{c}(\delta) \geqq 1 / \sqrt{\pi} \forall \delta>0$, with $v_{c}(\delta) \rightarrow 1^{+} / \sqrt{\pi}$ as $\delta \rightarrow 0^{+}$.

Proof. Follows directly from Lemmas 3.39-3.44.

The above theorem concludes our analysis of IVP1.

4. The initial value problem in $X<0$

In this section we consider the initial value problem,

$$
\begin{gathered}
\bar{u}_{X X}+\frac{1}{2} X \bar{u}_{X}+\left[\bar{u}^{p}-\frac{1}{1-p} \bar{u}\right]_{+}=0, \quad X<0, \\
\bar{u}(0)=(1-p)^{1 /(1-p)}+\delta, \quad \bar{u}_{X}(0)=-v \delta
\end{gathered}
$$

with $\delta, v \geqq 0$, which we henceforth refer to as IVP2. Again, we can make the following remark.

Remark 4.3. With $\delta=0$, IVP2 has the global solution $\bar{u}(X) \equiv(1-p)^{1 /(1-p)} \forall X \leqq 0$. It follows that this is unique $([8, \mathrm{Ch} .1$, Theorem 2.2$])$, from application of the local uniqueness theorem.

To proceed further we re-write IVP2 in terms of $\zeta=-X$,

$$
\begin{gathered}
\bar{u}_{\zeta \zeta}+\frac{1}{2} \zeta \bar{u}_{\zeta}+\left[\bar{u}^{p}-\frac{1}{1-p} \bar{u}\right]_{+}=0, \quad \zeta>0, \\
\bar{u}(0)=(1-p)^{1 /(1-p)}+\delta, \quad \bar{u}_{\zeta}(0)=v \delta
\end{gathered}
$$

which we refer to as $\overline{\text { IVP2 }}$. This now falls into the same class as IVP1 (with $v$ replaced by $-v$ ), and we have the following:

Theorem 4.6. For each $\delta>0$ and $v \geqq 0$, IVP2 has a unique solution in $X<0$. Moreover, this solution is monotone decreasing in $X$ with $\bar{u}(X) \rightarrow+\infty$ as $X \rightarrow-\infty$.

Proof. We work with the equivalent problem $\overline{\mathrm{IVP} 2}$ in $\zeta>0$, with solution $\bar{u}(\zeta)$. We define $\bar{u}_{l}(\zeta)$ and $\bar{u}^{l}(\zeta)$ as before, except we replace conditions $(3.6),(3.11)$ by $\bar{u}_{l \zeta}(0)=$ 
$\bar{u}_{\zeta}^{l}(0)=v \delta$. Similarly following the proofs of Propositions 3.9, 3.16, we readily establish that on any interval $\left[0, \zeta_{e}\right], \bar{u}_{l}(\zeta)$ and $\bar{u}^{-1}(\zeta)$ provide lower and upper bounds on $\bar{u}(\zeta)$ respectively. These $a$ priori bounds then enable existence and uniqueness for IVP2 to be established in $\left[0, \zeta_{e}\right]$ for any $\zeta_{e}>0$. Now, since $\bar{u}(0)>(1-p)^{1 /(1-p)}$ and $\bar{u}_{\zeta}(0) \geqq 0$, an examination of equation (4.4) establishes directly that $\bar{u}(\zeta)$ is monotone increasing in $\zeta>0$. Moreover, since $\bar{u}(\zeta) \geqq \bar{u}_{l}(\zeta)$ in $\zeta>0$, then $\bar{u}(\zeta) \rightarrow \infty$ as $\zeta \rightarrow \infty$, as required.

We are able to use the information that $\bar{u}(X) \rightarrow \infty$ as $X \rightarrow-\infty$ to obtain the asymptotic form,

$$
\bar{u}(X) \sim A(\delta, v)(-X)^{2 /(1-p)} \quad \text { as } \quad X \rightarrow-\infty,
$$

with $A(\delta, v)>0$ for any $\delta>0, v \geqq 0$. We can now return to the original problem BVP.

\section{The boundary value problem BVP}

We first return to $\overline{\mathrm{BVP}}$. Through Proposition 2.7, we observe that any solution, $U(X)$, to $\overline{\mathrm{BVP}}$ has $U(0)>(1-p)^{1 /(1-p)}$ and $U_{X}(0)<0$. Thus we may write for any solution to $\overline{\mathrm{BVP}}$,

$$
U(0)=(1-p)^{1 /(1-p)}+\delta, \quad U_{X}(0)=-v \delta,
$$

for some $\delta, v>0$. This leads us to:

Theorem 5.2. There is a bijection between solutions to $\overline{\mathrm{BVP}}$ and those pairs $(\delta, v) \in \mathbb{R}^{+} \times \mathbb{R}^{+}$for which IVP1 has a solution $\tilde{u}(X, v)$ with $\tilde{u}(X, v) \rightarrow(1-p)^{1 /(1-p)}$ as $X \rightarrow \infty$.

Proof. Let $S \subseteq \mathbb{R}^{+} \times \mathbb{R}^{+}$be defined by,

$S=\left\{(\delta, v):\right.$ IVPI has a solution at $(\delta, v)$ with $\tilde{u}(X, v) \rightarrow(1-p)^{1 /(1-p)}$ has $\left.X \rightarrow \infty\right\}$, and,

$$
B=\left\{\hat{u}: \mathbb{R} \rightarrow\left((1-p)^{1 /(1-p)}, \infty\right): \hat{u}(X) \text { is a solution of } \overline{\mathrm{BVP}}\right\}
$$

Now define the mapping $T: B \rightarrow S$ by $T[\hat{u}(X)]=(\delta, v)$, where,

$$
\delta=\hat{u}(0)-(1-p)^{1 /(1-p)}, \quad v=\frac{-\hat{u}_{X}(0)}{\left[\hat{u}(0)-(1-p)^{1 /(1-p)}\right]} .
$$

Clearly, $T$ is well-defined. We must now show that $T$ is one-one and onto.

(i) One-one

Suppose $\hat{u}_{1}(X)$ and $\hat{u}_{2}(X) \in B$ and $T\left[\hat{u}_{1}(X)\right]=T\left[\hat{u}_{2}(X)\right]$. Then, by definition of $T$, $\hat{u}_{1}(0)=\hat{u}_{2}(0)$ and $\hat{u}_{1 X}(0)=\hat{u}_{2 X}(0)$. Thus, in $X \geqq 0$, both $\hat{u}_{1}(X)$ and $\hat{u}_{2}(X)$ satisfy IVP1 with 
the same $v, \delta>0$ (see (5.1)). Uniqueness follows from Proposition 3.18 and Extension 3.20 , and so, $\hat{u}_{1}(X) \equiv \hat{u}_{2}(X)$ in $X \geqq 0$. Similarly, Theorem 4.6 shows that $\hat{u}_{1}(X) \equiv \hat{u}_{2}(X)$ in $X<0$. Hence $\hat{u}_{1}(X)=\hat{u}_{2}(X) \forall X \in \mathbb{R}$ and $T$ is one-one.

(ii) Onto

Let $(\hat{\delta}, \hat{v}) \in S$, then we define,

$$
\hat{u}(X)= \begin{cases}\tilde{u}(X, \hat{v}), & X \geqq 0 \\ \bar{u}(X, \hat{v}), & X<0 .\end{cases}
$$

Now, via Theorem 3.51, since $\tilde{u}(X, \hat{v}) \rightarrow(1-p)^{1 /(1-p)}$ as $X \rightarrow \infty$, then $\tilde{u}(X, \hat{v})$ is monotone decreasing in $X$ with $\tilde{u}(X, \hat{v})>(1-p)^{1 /(1-p)} \forall X>0$. Also, via Theorem 4.6, $\bar{u}(X, \hat{v})$ is monotone decreasing in $X<0$ with $\bar{u}(X, \hat{v}) \rightarrow \infty$ as $X \rightarrow-\infty$ and has asymptotic form (4.7). Therefore $\hat{u}(X) \in B$ and so $T$ is onto.

We note that $S=\left\{(\delta, v): v=v_{c}(\delta), \delta>0\right\}$, via Theorem 3.51 .

Remark 5.3. The correspondence of Theorem 5.2 relates solutions of $\overline{\mathrm{BVP}}$ uniquely to points in the positive quadrant of the $(\delta, v)$ plane.

Theorem 5.4. The set of solutions to $\overline{\mathrm{BVP}}$ consists of a one-parameter family, which can be parametrized by $\delta>0$. For each $\delta>0 \exists$ a unique solution to $\overline{\mathrm{BVP}}$ if and only if $\nu=v_{c}(\delta)$. Moreover, that solution can be constructed in terms of solutions to IVP1, 2 as,

$$
\hat{u}(X)= \begin{cases}\tilde{u}\left(X, v_{c}(\delta)\right), & X \geqq 0 \\ \bar{u}\left(X, v_{c}(\delta)\right), & X<0 .\end{cases}
$$

Proof. The proof follows from Theorem 5.2, 3.51.

Remark 5.6. From definition 5.5, we readily deduce that at any fixed $X \in \mathbb{R}, \hat{u}(X, \delta)$ is a continuous function of $\delta \geqq 0$. In addition we observe that with $\delta=0$ in (5.15) then $\hat{u}(X, 0) \equiv(1-p)^{1 /(1-p)} \forall X \in \mathbb{R}$, via Remarks 3.3, 4.3. Hence for fixed $X \in \mathbb{R} \hat{u}(X, \delta) \rightarrow$ $(1-p)^{1^{+} /(1-p)}$ as $\delta \rightarrow 0^{+}$.

In the remaining part of the paper, we relate solutions of $\overline{\mathrm{BVP}}$ to solutions of BVP. We begin with:

Proposition 5.7 The function $\chi(\delta) \equiv \delta v_{c}(\delta)$, for $\delta>0$, is non-decreasing. Moreover $\chi(\delta) \geqq(1 / \sqrt{\pi}) \delta$ in $\delta>0$, and $\chi(\delta) \sim(1 / \sqrt{\pi}) \delta$ as $\delta \rightarrow 0^{+}$.

Proof. Suppose that $\delta_{1}>\delta_{0}>0$ and that $\chi\left(\delta_{1}\right)<\chi\left(\delta_{0}\right)$. Hence $\delta_{1} v_{1}<\delta_{0} v_{0}$, where $v_{1}=v_{c}\left(\delta_{1}\right), v_{0}=v_{c}\left(\delta_{0}\right)$. Then, via the nonlinear comparison theorem, [23], the solution of IVP1 with $\delta=\delta_{0}, v=\delta_{1} v_{1} / \delta_{0}$ has $\tilde{u}(X) \rightarrow(1-p)^{1 /(1-p)}$ as $X \rightarrow \infty$ (as it is bounded above by the solution to IVP1 with $\delta=\delta_{1}, v=v_{1}$ and bounded below by the solution to IVP1 
with $\left.\delta=\delta_{0}, v=v_{0}\right)$. Thus $\delta_{1} v_{1} / \delta_{0} \in J_{\delta_{0}}$. But $v_{0} \in J_{\delta_{0}}$ and $v_{0} \neq \delta_{1} v_{1} / \delta_{0}$, which contradicts Lemmas $3.43,3.44$. Therefore $\chi\left(\delta_{1}\right) \geqq \chi\left(\delta_{0}\right)$ and the results follows. The final part follows from Theorem 3.51 .

The family of solutions to $\overline{\mathrm{BVP}},(5.5)$, have the following behaviour as $X \rightarrow-\infty$,

$$
\hat{u}(X, \delta) \sim \Phi(\delta)(-X)^{2 /(1-p)} \quad \text { as } \quad X \rightarrow-\infty
$$

via (4.7), with $\Phi(\delta)=A\left(\delta, v_{c}(\delta)\right)$, and $\Phi(\delta)>0$ for all $\delta>0$. We can establish the following properties of $\Phi(\delta)$ :

Proposition 5.9. In $\delta>0, \Phi(\delta)$ is monotone increasing with $\Phi(\delta) \rightarrow 0^{+}$as $\delta \rightarrow 0^{+}$and $\Phi(\delta) \rightarrow \infty$ as $\delta \rightarrow \infty$.

Proof. Continuous dependence of $\hat{u}(X, \delta)$ on $\delta$ (initial conditions) establishes the continuity of $\Phi(\delta)$ with $\delta>0$. Also at $\delta=0$ (via Remark 5.6), $\hat{u}(X, \delta) \equiv 0 \forall X \in \mathbb{R}$, and continuity of $\hat{u}(X, \delta)$ on $\delta$ for fixed $X$ at $\delta=0$ (Remark 5.6) requires $\lim _{\delta \rightarrow 0^{+}} \Phi(\delta)=0$. Now, for $\delta_{1}>\delta_{0}>0$ we have (via the nonlinear comparison theorem, [23], in $X<0$, and Proposition 5.7) $\hat{u}\left(X, \delta_{1}\right)>\hat{u}\left(X, \delta_{0}\right)$ and $\left[-\hat{u}_{X}\left(X, \delta_{1}\right)\right] \geqq\left[-\hat{u}_{X}\left(X, \delta_{0}\right)\right] \forall X<0$. Thus, via (5.8) $\Phi\left(\delta_{1}\right)>\Phi\left(\delta_{0}\right)$, as required. We next show that for a given $\delta>0, \hat{u}_{X}(X, \delta)$ is monotone decreasing in $X<0$. Suppose that $\hat{u}_{X}(X, \delta)$ has a turning point in $X<0$, at $X=X_{T}$ say, then $\hat{u}_{X X}\left(X_{T}, \delta\right)=0$, and so, via equations $(5.5),(4.1), \hat{u}_{X}\left(X_{T}, \delta\right)=$ $X^{T-1}\left[(1 /(1-p)) \hat{u}\left(X_{T}, \delta\right)-\hat{u}^{p}\left(X_{T}, \delta\right)\right]_{+}>0$ as $\hat{u}\left(X_{T}, \delta\right)>(1-p)^{1 /(1-p)}$. However, via Theorem 4.6 and $(5.5), \hat{u}_{X}\left(X_{T}, \delta\right)<0$, which gives a contradiction. Hence $\hat{u}_{X}(X, \delta)$ is monotone in $X<0$, and is monotone decreasing via (5.8). It now follows directly from Proposition 4.7 (noting that $\hat{u}_{X}(0, \delta)=-\chi(\delta)$ ) and (5.8) that $\Phi(\delta) \rightarrow \infty$ as $\delta \rightarrow \infty$. This completes the proof.

Finally we are able to return to our original boundary value problem BVP given by (1.8)-(1.10). We have:

Theorem 5.10. For each $g_{\sigma}>0$, BVP has a unique solution. Moreover that solution is given by $V=\hat{u}(X, \delta)$, where $\delta$ is the unique, positive, root of the equation $\Phi(\delta)-g_{\sigma}=0$.

Proof. The proof follows directly from properties of $\hat{u}(X ; \delta), \delta>0$.

\section{REFERENCES}

1. C. Bande and H. A. Levine, On the existence and non-existence of global solutions of reaction-diffusion equations in sectorial domains, Trans. Amer. Math. Soc. 665 (1989), 595-624.

2. C. Bande and I. Stakgold, The formation of the dead core in parabolic reaction-diffusion problems, Trans. Amer. Math. Soc. 286 (1984), 275-293.

3. H. Berestycki, P. L. Lions and L. A. Peletier, An ODE approach to the existence of positive solutions in for semilinear problems in $\mathbb{R}^{N}$, Indiana Univ. Math. J. 30 (1981), 141-157.

4. J. Billingham and D. J. Needham, A note on the properties of travelling wave solutions arising in cubic autocatalysis, Dynamic Stability Systems 6 (1991), 33-49.

5. J. Billingham and D. J. Needham, The development of travelling waves in quadratic and cubic autocatalysis with unequal diffussion rates I. Permanent form travelling waves, Philos. Trans. Roy. Soc. London Ser A 334 (1991), 1-24. 
6. J. Billingham and D. J. Needham, The development of travelling waves in quadratic and cubic autocatalysis with unequal diffusion rates II. An initial value problem with an immobilized or nearly immobilized autocatalyst, Philos. Trans. Roy. Soc. London Ser A 336 (1991), 4967-5539.

7. J. Billingham and D. J. Needham, The development of travelling waves in quadratic and cubic autocatalysis with unequal diffusion rates III. Large time development in quadratic autocatalysis, Quart. Appl. Math. 50 (1992), 343-372.

8. E. A. Coddington and N. Levinson, The theory of ordinary differential equations (McGrawHill, New York, 1955).

9. M. EscoBedo and E. ZuA ZuA, Self-similar solutions for a convection-diffusion equation with absorption in $\mathbb{R}^{N}$, Israel J. Math. 74 (1991), 47-64.

10. H. Fustr, On the blowing up of solutions of the Cauchy problem for $u_{t}=\Delta u+u^{1+\alpha}, J$. Fac. Sci. Univ. Tokyo Sect. A Math. 16 (1966), 105-113.

11. P. Gray, J. H. Merkin, D. J. Needham and S. K. Scott, The development of travelling waves in a simple isothermal chemical system III. Cubic and mixed autocatalysis, Proc. Roy. Soc. London Ser. A 430 (1990), 509-524.

12. R. E. Grundy and L. A. Peletier, Short time behaviour of a singulation solution to the heat equation with absorption, Proc. Roy. Soc. Edinburgh 107A (1987), 271-288.

13. M. W. Hirsch and S. SMALE, Differential equations, dynamical systems and linear algebra (Academic Press, New York, 1974).

14. A. C. King and D. J. Needham, On a singular initial boundary value problem for a reaction-diffusion equation arising from a simple model of isothermal chemical autocatalysis, Proc. Roy. Soc. London Ser A 437 (1992), 657-671.

15. H. A. Levine, The role of critical exponents in blowup theorems, SIAM Rev. 32 (1990), 262-288.

16. J. H. Merkin and D. J. Needham, Propagating reaction-diffusion wabes in a simple isothermal quadratic chemical system, J. Engrg. Math. 23 (1989), 343-356.

17. J. H. Merkin and D. J. Needham, The development of travelling waves in a simple isothermal chemical system II. Cubic autocatalysis with quadratic decay and with linear decay, Proc. Roy. Soc. London Ser A 430 (1990), 315-345.

18. J. H. Merkin and D. J. Needham, The development of travelling waves in a simple isothermal chemical system IV. Quadratic autocatalysis with quadratic decay, Proc. Roy. Soc. London Ser A 434 (1991), 531-554.

19. J. H. Merkin D. J. Needham and S.K. Scott, The development of travelling waves in a simple isothermal chemical system I. Quadratic autocatalysis with linear decay, Proc. Roy. Soc. London Ser A 424 (1989), 187-209.

20. D. J. NeEDHAM, On the global existence of solutions to a singular parabolic equation arising from the study of autocatalytic chemical kinetics, Z. Angew. Math. Phys. 43 (1992), 471-480.

21. D. J. Needham and J. H. Merkin, The development of travelling waves in a simple isothermal chemical system with general orders of autocatalysis and decay, Proc. Roy. Soc. London Ser A 337 (1991), 261-274.

22. L. A. Peletier and J. Serrin, Uniqueness of positive solutions of semilinear equations in $\mathbb{R}^{N}$, Arch. Rational Mech. Anal. 81 (1983), 181-197.

23. M. H. Protter and H. F. Weingerger, Maximum principles in differential equations (Prentice-Hall, Engelwood Cliffs, New Jersey, 1967). 
24. F. B. WeIsSLer, Existence and non-existence of global solutions for a semilinear heat equation, Israel J. Math. 38 (1981), 29-40.

School of Mathematics

UNIVERSITY OF EAST ANGLIA

NoRWICH NR4 7TJ
Department of Mathematics

UNIVERSITY OF KeELE

KEELE

STAFFordShIRE ST5 5BG 